\title{
STATISTICAL ANALYSIS OF PERIOD-ECCENTRICITY DISTRIBUTION FOR LARGE SAMPLES OF CLOSE BINARIES FROM KEPLER, COROT AND SB9 DATABASES
}

\author{
J. Sztakovics ${ }^{1,2}$, E. Forgács-Dajka ${ }^{1}$, T. Borkovits ${ }^{3}$, T. Hajdu ${ }^{1,2}$ \\ and G. Marschalkó 2,3
}

\begin{abstract}
Period-eccentricity distribution of eccentric eclipsing binaries (eEB) has inevitable importance in the study of the different kinds of tidal circularization (and synchronization) processes. Recent space missions provide a large amount of continuous and precise light curves, which enable us to determine several accurate stellar and dynamical parameters for thousands of freshly discovered eEBs. For statistical analysis we developed a fast, automated, although approximating method for determining eccentricity and argument of periastron from the displacement of the secondary minima and the difference of primary and secondary eclipse durations for large samples of eclipsing lightcurves. Applying this method to several hundreds of recently discovered eEBs in the fields of the primordial and secondary Kepler and also the CoRoT missions, we present a comprehensive statistical analysis of the period-eccentricity relation for different subgroups of these double star systems.
\end{abstract}

\section{Introduction}

The study of tides has a long and amazing history. It involves an interesting combination of celestial mechanics, which is non-trivial but can be explored, and stellar structure and evolution, which involves some deeper physical issues. Binary stars can contribute to investigating these processes. For example, in tight binaries tides can synchronize the spin of the stars with the orbit and circularize an eccentric orbit as the binary tends towards an equilibrium state of minimum energy. One interesting aspect of tidal circularization theory is that the dissipation rate is a very

\footnotetext{
${ }^{1}$ Eötvös University, Department of Astronomy, H-1518 Budapest, P.O. Box 32, Hungary

${ }^{2}$ Wigner Research Centre for Physics of the H.A.S., H-1525 Budapest, P.O. Box 49, Hungary

${ }^{3}$ Baja Astronomical Observatory of Szeged Univ., H-6500 Baja, Szegedi út kt. 766, Hungary
} 
strong function of the orbital semi-major axis, and thereby of the orbital period. Many studies attemped to determine the so-called cut-off period (see e.g. Mayor \& Mermilliod 1984), which characterizes the transition between mainly-circular and mainly-eccentric orbits. Another interesting aspect of the theory is that the circularization timescale is predicted to depend strongly on stellar type. The two tidal dissipation processes that have received most attention so far are the turbulent friction acting on the equilibrium tide, which was first described in the $60 \mathrm{~s}$ (Zahn 1966; Zahn 1977), and the radiative damping of the dynamical tide, identified in the 70s (Zahn 1977). These processes operate respectively in convection and radiation zones, so stars with thick convective envelope are expected to experience more rapid tidal dissipation than stars with radiative envelope. Accordingly, we adopted the definition of cool $\left(T_{\text {eff }}<7000 \mathrm{~K}\right)$ and hot $\left(T_{\text {eff }}>7000 \mathrm{~K}\right)$ stars (see e.g. Torres et al. 2010) which corresponds to stars with thick convective or mainly radiative exterior.

Recent space missions (Kepler, K2, CoRoT) provides a large number of continuous and precise photometric data, which enable us to determine several accurate stellar and dynamical paramters for thousands of newly discovered eclipsing binaries $(\mathrm{EBs})$. In order to achieve fast data processing and to compile statistics, we developed a fast, automated, although approximating method on GPU for determining eccentricity and argument of periastron from the phases of the durations of the primary and secondary eclipses, and from the phase of the secondary eclipse.

\section{Data and method}

For our investigations, we used the data from the Villanova Kepler EB Catalog ${ }^{1}$ (Prša et al. 2011) and Archive of the CoRoT Data Center Release 13 (COROT Team 2012). We collected the preprocessed lightcurves of all detached, so-called Algol-type eclipsing binaries (those systems for which the morphology parameter is $<0.5$, see Matijevič et al. 2012). First, we checked or (re)determined the orbital period by the Lomb-Scargle periodogram and/or Eclipse Timing Variation (ETV) method, so with the specified period we folded, binned and averaged the light curve for each system. Second, we localized the primary and secondary minima and the phases of theme. With this end in view, we fitted only the minima with the following function:

$$
f\left(t_{i}\right)=c_{0}+c_{1}\left(1-\left\{1-\exp \left[\cosh \left(\frac{t_{i}-t_{0}}{d}\right)\right]^{\Gamma}\right\}\right)
$$

where $c_{0}$ and $c_{i}$ are additive and multiplicative constants, $t_{0}$ is the mid-time of the eclipse, $t_{i}$ is the independent variable, $d$ is the width of the minimum, and $\Gamma$ defines the pointedness of the eclipse (Mikulášek 2015). This formalism is only a phenomenological description of an eclipse and gives a mathematical determination of the minima.

\footnotetext{
${ }^{1}$ http://keplerebs.villanova.edu
} 

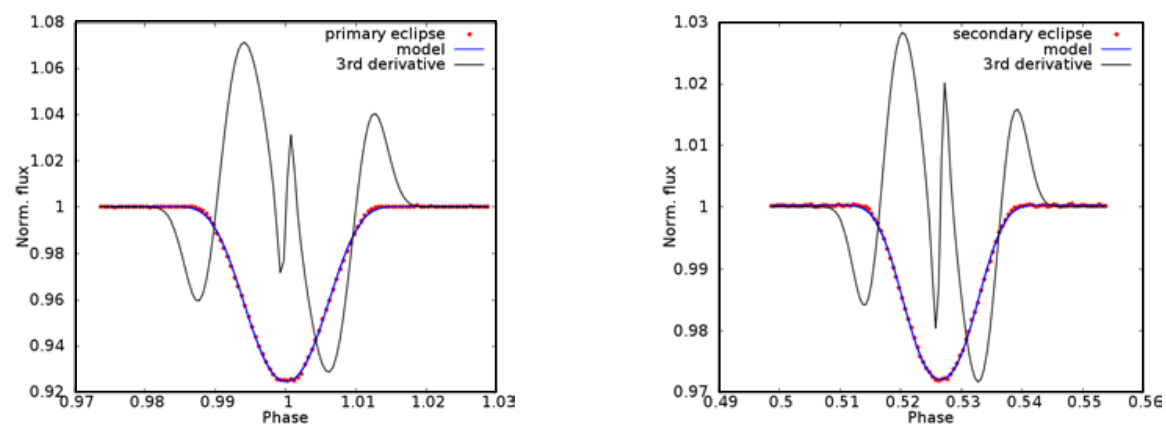

Fig. 1. Folded and binned primary and secondary eclipse data points of KIC01026032 from the Kepler sample (red dots) with the fitted model (blue line), and the third derivative (black line). The derivative's local extrema approximate the contact phases of the system.

We calculated the third derivative of the fitted functions, because we found that the derivatives' local extrema are good markers of the first and last contact phases $\left(t_{1}, t_{4}\right)$ of the binary's orbit. From these identified quantities, the eccentricity and the argument of periastron are calculated using the following equations:

$$
\begin{gathered}
e \sin \omega \approx \frac{t_{s 14}-t_{p 14}}{t_{s 14}+t_{p 14}} \\
e \cos \omega \approx(0.5-\delta \varphi) \frac{\pi}{2},
\end{gathered}
$$

where $t_{p 14}, t_{s 14}$ are the durations of the primary and secondary eclipse and $\delta \varphi$ is the difference in phase between the primary and secondary eclipses (Kopal 1959). In both cases we assume that the inclination is $90^{\circ}$, so the dependence of these quantities on the inclination is neglected, hence this approximation can be applied to detached, Algol-type systems. Based on Equation (2.2) and Equation (2.3) the binary's orbital eccentricity and the argumentum of periastron can be easily calculated.

\section{Results}

Applying this method to all these systems, we derived eccentricities and arguments of periastron for 724 Kepler and 345 CoRoT detached binaries. As we mentioned in Section 1, to investigate the tidal processes and circularization timescales we defined the cool and the hot systems based on the effective temperature of the primary star. These physical parameters are obtained from Armstrong et al. (2014) for Kepler data, but for CoRoT data only the spectral classes are available (COROT Team 2012), from which we assume the temperatures. To achieve a more precise statistical analysis we expand our sample with the SB9 Catalog 

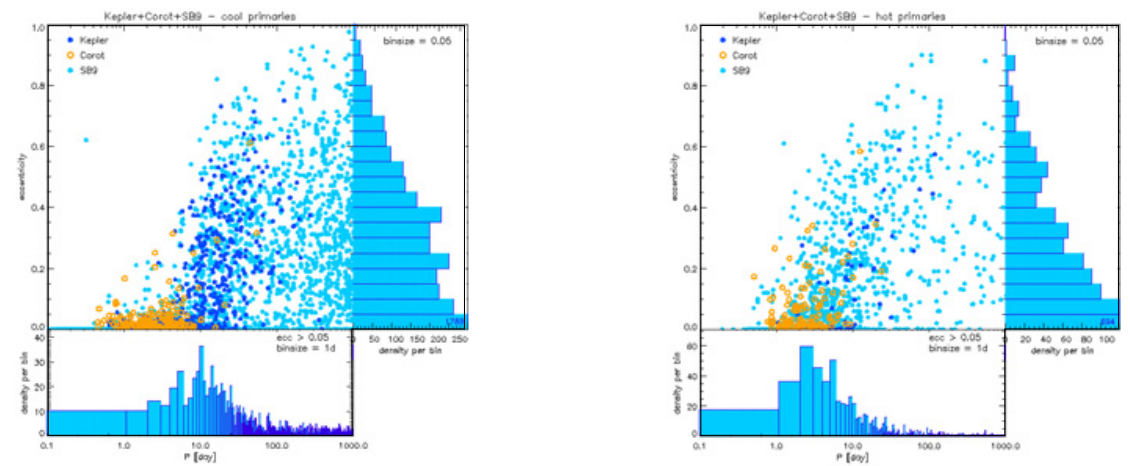

Fig. 2. Period-eccentricity diagrams for all systems from the Kepler (blue circles), CoRoT (yellow circles) and SB9 (light blue circles) with cool and hot primaries respectively.
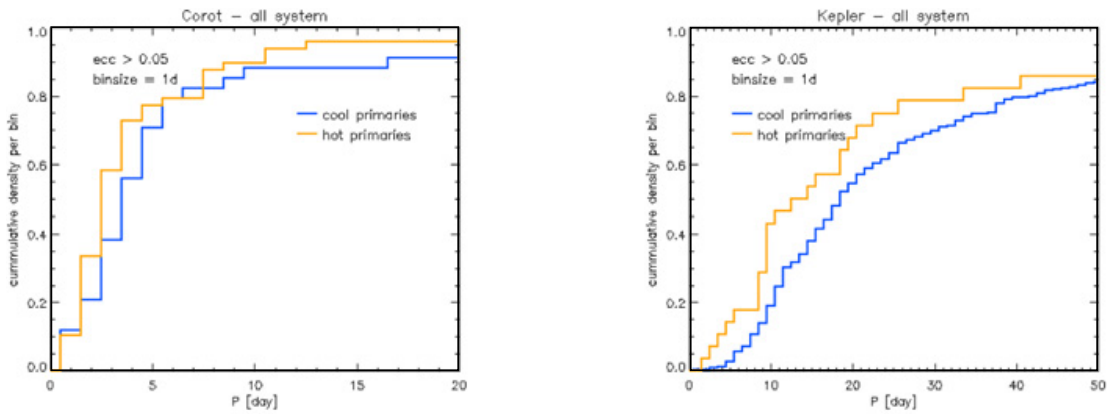

Fig. 3. Cumulative density diagrams for all systems from the Kepler (right panel) and the CoRoT with hot (orange line) and cool (blue line) primaries.

(Pourbaix et al. 2004). In this database of spectroscopic binaries orbital parameters such as eccentricity and period are determined from radial velocity measurements.

The results of our survey can be seen in Figure 2, where we plot periodeccentricity diagrams for all systems and histograms for both period and eccentricity. We separate the systems based on hot and cool primary to examine the difference between the tidal processes and timescales related to them. We found the followings: (i) the mean eccentricity for systems $e>0.05-$ not including the circular orbits, since the fully circularized systems are not relevant to our study - is $e_{\text {hot }}=0.27$ and $e_{\text {cool }}=0.27$ for Kepler and $e_{\text {hot }}=0.15$ and $e_{\text {cool }}=0.13$ for CoRoT sample. The mean values from CoRoT are remarkably smaller than we can expect from other data, because the observation time is significantly shorter $(\approx 150$ days) than, for example, for the 4-year-long Kepler mission, so only the systems with shorter periods (lower eccentricities) are observed. Based on the richest sample, the SB9 Catalog, these values are $e_{\text {hot }}=0.31$ and $e_{\text {cool }}=0.37$. (ii) In case of cool 
systems, we found peaks in the histograms of period and eccenticity, too. These values are around $P \approx 10^{d}, e \approx 0.2$ and $e \approx 0.4$, but the origin of these peaks is not clear yet, further investigations are needed. (iii) In the cumulative density diagrams (see Fig. 3) we can determine the cut-off period, which characterizes the transition between circular and eccentric orbits. It can be seen that this period is about $P \approx 2^{d}$ for systems with hot primary and $P \approx 4-5^{d}$ for systems with cool primary. These results are in a good agreement with the theory of Zahn (see, e.g. Zahn 1966, 1977), so the systems where the primary star has thick convective envelope circularize faster on account of the more effective tidal dissipation. These results are confirmed only by the Kepler data, while the CoRoT data as stated above are not sufficiently populated to verify it.

J.Sz., E.F.-D. and T.B. thanks for the organizers their kindest invitation and hospitality. The conference participation of J.Sz. was subsidised by the Talented Student Program of Eötvös Loránd University, Budapest. This project has been supported by the Hungarian OTKA Grant K113117.

\section{References}

Armstrong, D.J., Gómez Maqueo Chew, Y., Faedi, F., \& Pollacco, D., 2014, MNRAS, 437,3473

COROT Team, 2012, VizieR Online Data Catalog, 1

Kopal, Z., 1959, The International Astrophysics Series, London: Chapman \& Hall, 1959

Matijevič, G., Prša, A., Orosz, J.A., et al., 2012, AJ, 143, 123

Mayor, M., \& Mermilliod, J.C., 1984, Observational Tests of the Stellar Evolution Theory, 105,411

Mikulášek, Z., 2015, A\&A, 584, A8

Pourbaix, D., Tokovinin, A.A., Batten, A.H., et al., 2004, A\&A, 424, 727

Prša, A., Batalha, N., Slawson, R.W., et al., 2011, AJ, 141, 83

Torres, G., Andersen, J., \& Giménez, A., 2010, Astron, Astrophys. Rev., 18, 67

Zahn, J.P., 1966, Annales d'Astrophysique, 29, 313

Zahn, J.-P., 1977, A\&A, 57, 383 


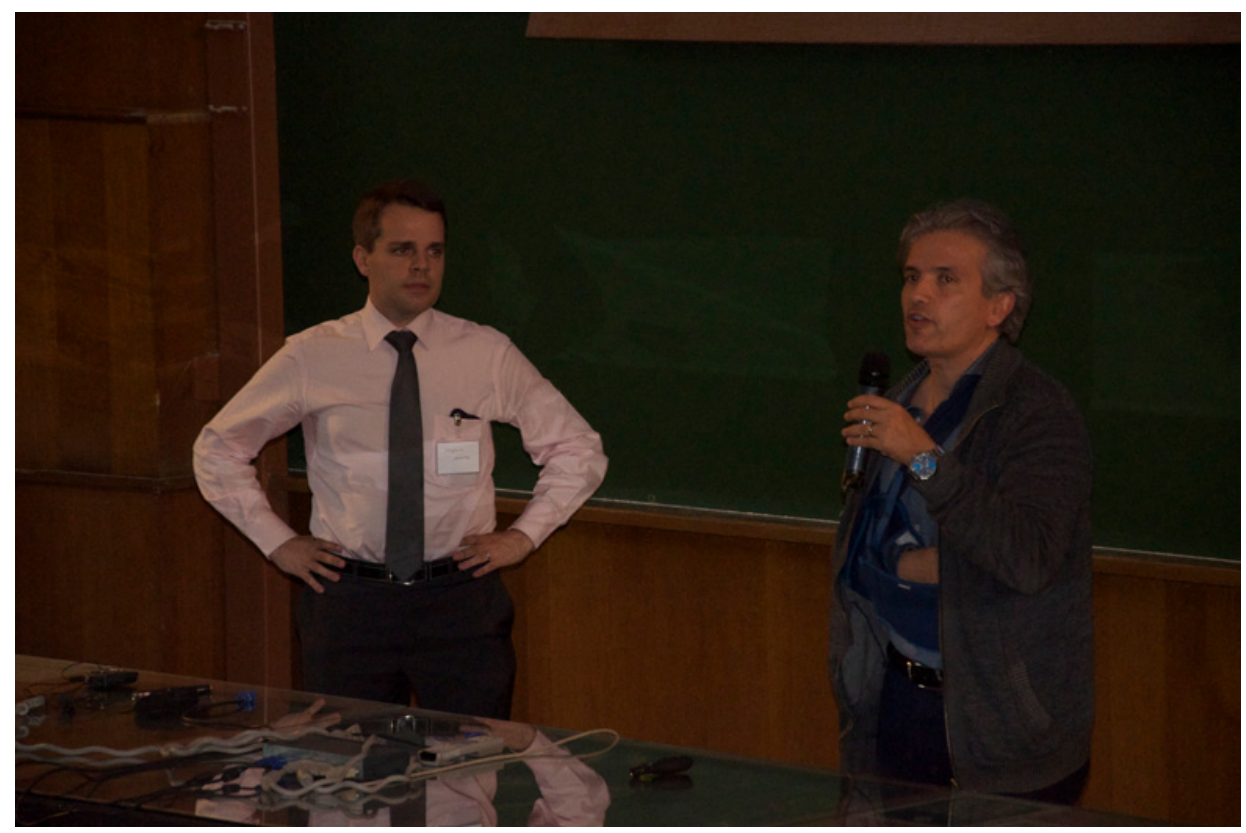

Stéphane Mathis and Allan Sacha Brun

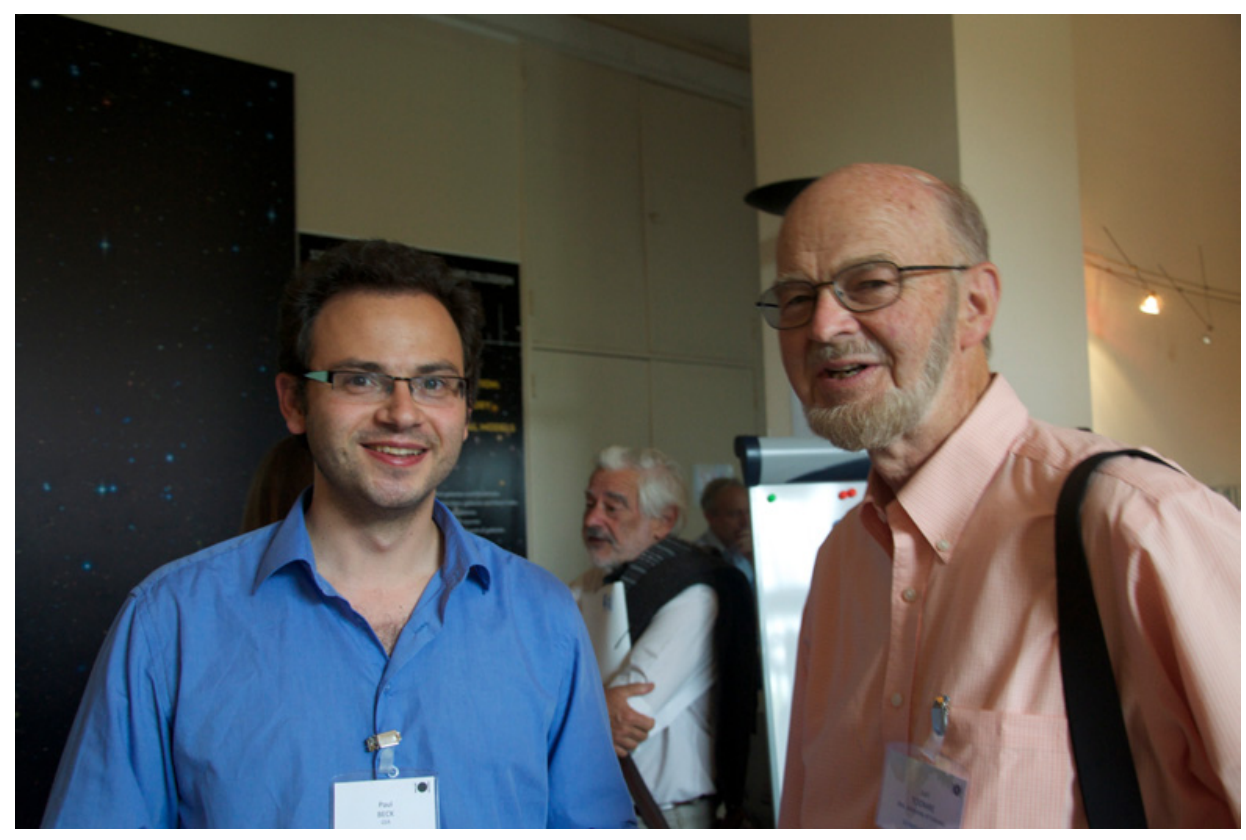

Paul Beck and Juri Toomre 


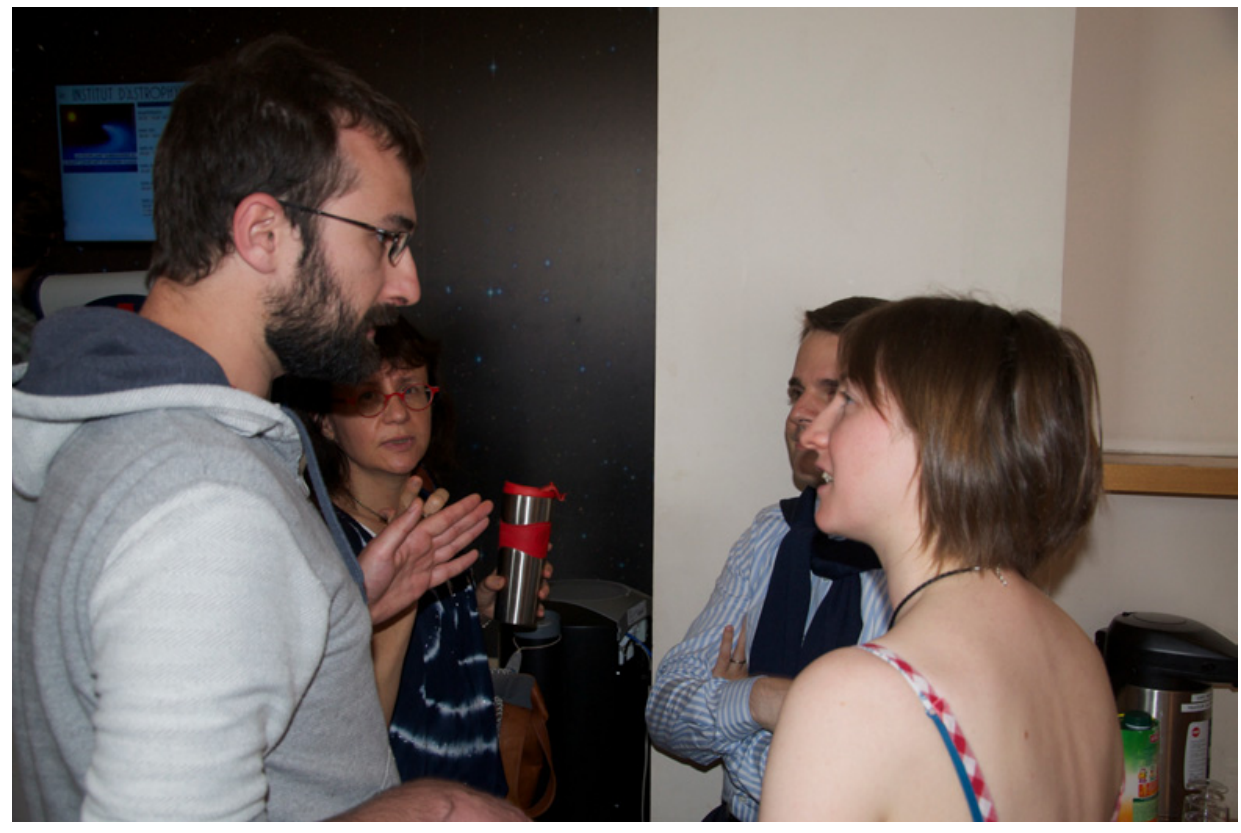

Florian Gallet, Emeline Bolmont, Corinne Charbonnel and Stéphane Mathis 